Instituto Internacional de Investigación y Desarrollo Tecnológico Educativo INDTEC, C.A.

DOI: https://doi.org/10.29394/Scientific.issn.2542-2987.2020.5.E.2.48-69

OAI-PMH: http://www.indteca.com/ojs/index.php/Revista Scientific/oai

Artículo Original / Original Article

\title{
Adaptación social a la comunidad educativa de niños venezolanos en situación de movilidad humana
}

Autoras: Elizabeth Alexandra Dávila Cueva Universidad Internacional SEK, UISEK eadavila.mpp@uisek.edu.ec

Quito, Ecuador

https://orcid.org/0000-0003-3679-946X

Mirian Isabel González Chicaiza

Universidad Internacional SEK, UISEK migonzalez.mpp@uisek.edu.ec

Quito, Ecuador

https://orcid.org/0000-0001-6222-6089

Diana Cristina Castellanos Vela Universidad Internacional SEK, UISEK dianacastellanosv@gmail.com

Quito, Ecuador https://orcid.org/0000-0002-6651-6857

Janethcia del Rocío Játiva Morillo Universidad Internacional SEK, UISEK rocio.jativa@uisek.edu.ec

Quito, Ecuador

https://orcid.org/0000-0001-7235-1924

\section{Resumen}

El presente artículo tiene como objetivo indagar sobre la relación de la dinámica familiar con la resolución del duelo en los procesos de migración o movilidad humana, y como estos aspectos se relacionan en la adaptación social de niños y niñas al sistema educativo del país de destino. La investigación tiene un enfoque cualitativo, de tipo descriptiva, en la que participaron tres niños de nacionalidad venezolana, que fueron derivados por sus escuelas a centros psicológicos, por presentar dificultades en las relaciones que establecen con sus pares y docentes. Tras aplicarse los instrumentos cualitativos (entrevistas semiestructuradas y aplicación del genograma) se obtuvieron resultados vinculados a las experiencias del proceso de movilidad humana, su dinámica familiar, adaptación social, inclusión escolar en el país de destino y participación del núcleo familiar al nuevo contexto. Estos resultados contribuyeron a resaltar la importancia de trabajar con las familias en los procesos de elaboración del duelo y generar programas específicos para el sistema educativo con el propósito de implementar acciones que lo conviertan en un entorno favorable, que garantice el acceso a la educación y posibilite la inclusión y adaptación social de niñas y niños en situación de movilidad humana, en entornos seguros y amigables.

Palabras clave: adaptación social; migración; psicoterapia.

Cómo citar este artículo:

Dávila, E., González, M., Castellanos, D., \& Játiva, J. (2020). Adaptación social a la comunidad educativa de niños venezolanos en situación de movilidad humana. Revista Scientific, 5(Ed. Esp.), 48-69, e-ISSN: 2542-2987. Recuperado de: https://doi.org/10.29394/Scientific.issn.2542$\underline{2987.2020 .5 . E .2 .48-69}$

Fecha de Recepción: 02-04-2020
Fecha de Aceptación: 29-06-2020
Fecha de Publicación: 05-08-2020 
OAI-PMH: http://www.indteca.com/ojs/index.php/Revista_Scientific/oai

Artículo Original / Original Article

\title{
Social adaptation to the educational community of Venezuelan children in situations of human mobility
}

\begin{abstract}
This article aims to investigate the relationship of family dynamics with the resolution of mourning in the processes of migration or human mobility, and how these aspects are related to the social adaptation of boys and girls to the educational system of the destination country. The research has a qualitative, descriptive approach, in which three Venezuelan children participated, who were referred by their schools to psychological centers, for presenting difficulties in the relationships they establish with their peers and teachers. After applying the qualitative instruments (semi-structured interviews and application of the genogram), results were obtained related to the experiences of the human mobility process, its family dynamics, social adaptation, school inclusion in the destination country and participation of the family nucleus in the new context. These results contributed to highlight the importance of working with families in the processes of preparing grief and generating specific programs for the educational system in order to implement actions that make it a favorable environment, which guarantees access to education and enables the inclusion and social adaptation of girls and boys in situations of human mobility, in safe and friendly environments.
\end{abstract}

Keywords: student adjustment; migration; psychotherapy.

\footnotetext{
How to cite this article:

Dávila, E., González, M., Castellanos, D., \& Játiva, J. (2020). Social adaptation to the educational community of Venezuelan children in situations of human mobility. Revista Scientific, 5(Ed. Esp.), 48-69, e-ISSN: 2542-2987. Recovered from: https://doi.org/10.29394/Scientific.issn.25422987.2020.5.E.2.48-69
}

Date Received:

02-04-2020
Date Acceptance: 29-06-2020
Date Publication: 05-08-2020 


\section{Introducción}

En la actualidad, Venezuela se encuentra sumergida en una crisis social y esto conllevó procesos de migración sucesiva de una parte de su población. Para Ceja, et al. (2020): entre los países a los que sus habitantes se trasladan está Ecuador, el tercer país con mayor población venezolana en América.

La Organización Internacional para las Migraciones (OIM) precisa que la movilidad humana consiste en el ejercicio del derecho a la libre circulación que todos los individuos poseen. De acuerdo con Sánchez (2018): este desplazamiento puede ser una decisión voluntaria, también puede deberse a factores externos que se tornan amenazantes para su existencia, obligando a la persona a dejar su país de origen

En este sentido, Coronel (2013), expone que: esta situación de movilización, traslado y arribo a un lugar diferente, genera cambios en el ámbito psicológico de las personas y las sensaciones que se experimentan. Las sensaciones, como miedo, soledad y nostalgia, pueden dificultar la interacción con las personas en el país de llegada. El Colegio Oficial de Psicólogos de Madrid (2016): señala que, es necesario dar atención a estas consecuencias psicológicas, así como a las dificultades sociales, culturales y económicas que surgen producto de la migración.

Junto a las dificultades propias de este proceso migratorio se encuentra la pérdida de los vínculos que han establecido las personas con su nación y con los sujetos que los vieron crecer. Esto desencadena procesos de duelo, una respuesta emocional que experimenta el ser humano cuando pierde algo o a alguien. Por lo general, el fenómeno de la movilidad humana es estudiado desde diferentes ámbitos, pero no siempre se contempla a la persona, al ser individual, no se considera el dolor que experimenta, sus miedos, sus pérdidas, la necesidad de la elaboración del duelo que surge con el reconocimiento de la pérdida de todo aquello que le resultaba conocido y habitual, la aceptación de la realidad y la reorganización de la vida en función del cambio. Según 
González (2005a): el acercarse a la realidad de la persona en situación de movilidad humana desde el fenómeno del duelo, permite visibilizar los problemas que experimenta la persona en su proceso de adaptación, de integrar lo nuevo y de lo dejado atrás.

Un grupo vulnerable y en el que la situación de movilidad humana genera mayor incomprensión, es el de las y los niños, porque sus esferas personal, familiar y social se ven alteradas y enfrentan, al igual que en los adultos, pérdidas significativas al abandonar su país de origen. En conformidad con Atxotegui (2000): la elaboración del duelo en los niños en situación de movilidad humana, se constituye en una parte fundamental del contacto creativo y adaptativo, base del equilibrio psicológico del ser humano.

Dentro del sistema educativo nacional, la escuela aborda edades situadas entre 6 y 12 años. A nivel de desarrollo humano, se identifica que este rango etario atraviesa las etapas identificadas como niñez intermedia y niñez tardía. Siguiendo a Papalia, Wendkos y Duskin (2009): durante estas etapas de desarrollo, las relaciones interpersonales se incrementan y las relaciones externas a aquellas del grupo familiar son las que favorecen el desarrollo personal y social de niñas y niños.

En virtud de esta problemática, la presente investigación plantea la siguiente pregunta: ¿Cómo afecta la situación de movilidad humana en el proceso de adaptación social en niñas y niños venezolanos en el sistema educativo ecuatoriano? En este sentido, se plantea la siguiente hipótesis: En niñas y niños en situación de movilidad humana, la dinámica familiar favorece la resolución del duelo y, por tanto, el proceso de adaptación social al sistema educativo.

Ahora bien, el proceso de adaptación a un nuevo contexto por parte de los niños en situación de movilidad humana y la elaboración del duelo por las pérdidas emocionales, debe contemplar a la familia; pues, además, se considera que las conductas adaptativas de la familia ante los cambios y la 
nueva realidad y el proceso de elaboración de lo que ha quedado atrás se transmite, influye y moldea la personalidad de los niños. Mencionando a González (2005b): la manera en la que las familias elaboren el respectivo duelo ejercerá una marcada influencia sobre las demás generaciones y sus propios procesos de resolución del duelo, así como en el proceso de adaptación social.

El integrar a la familia para el trabajo en los procesos de adaptación social y elaboración del duelo, conforme al modelo de la terapia familiar sistémica, implica tomar en cuenta la estructura y el funcionamiento familiar, es decir quiénes componen este sistema familiar y cómo interactúan los miembros de dicha familia. Para esto, Suarez (2010): presenta que este modelo psicológico plantea el genograma como un instrumento valioso y útil para recolectar la información sobre el sistema familiar y mismo que se define como una representación gráfica que permite registrar información sobre su estructura y las relaciones de sus miembros.

Otro de los sistemas importantes es la escuela, sobre todo por la etapa de desarrollo de niñas y niños. En atención a Kreuz, Casas, Aguilar y Carbó (2009): la escuela, en conjunto con la familia, juega un papel vital para apoyar a los niños en situación de movilidad humana en su proceso de adaptación social y elaboración del duelo, con el objetivo de prevenir problemas que podrían derivar en consecuencias psicológicas que afecten el desarrollo de esta población.

La escuela se constituye en uno de los principales agentes socializadores que permite la adquisición de valores y normas, que ayudan al niño a integrarse a la sociedad y vivir en grupo. Además, Hernández (2019): articula que al ser un contexto que integra a diversas personas, ofrece experiencias sociales variadas que favorecen el desarrollo social y de la personalidad del niño

Organismos internacionales con agencias en Ecuador, como el Alto 
Comisionado de las Naciones Unidas para los Refugiados (ACNUR) y el Fondo para las Naciones Unidas para la Infancia (UNICEF), han coordinado con las autoridades nacionales una serie acciones para la protección de la población infantil y adolescente en situación de movilidad humana, abogando para que las y los niños que migran sean protegidos y sus derechos respetados.

Desde la psicoterapia, uno de los objetivos es la prevención de problemáticas que afecten la salud mental de poblaciones en situación de vulnerabilidad, como es el caso de quienes se encuentran en condiciones de movilidad humana, y, de requerirse, brindar la atención clínica para lograr un proceso de adaptación social y elaboración del duelo de manera adecuada. Al constituirse la escuela como un sistema que posibilita la socialización e interacción directa con los otros, esta proporciona, por un lado, gran cantidad de información al psicoterapeuta sobre el proceso de elaboración del duelo, sobre los procesos de adaptación social que puedan presentar los niños en situación de movilidad humana y cómo se manifiestan conductualmente cualquier tipo de dificultad. Por otra parte, la escuela se constituye en un espacio propicio para generar estrategias de prevención y atención oportuna de dichas dificultades.

Esto permite que, desde los espacios de atención psicológica, se pueda realizar el abordaje oportuno y partiendo desde lo preventivo, considerando al niño como miembro activo, dinámico de distintos sistemas, en este caso de la familia y de la escuela.

En este sentido, el presente estudio pretende demostrar que un adecuado proceso de elaboración del duelo puede aportar de manera positiva al proceso de adaptación de niños y niñas en situación de movilidad humana a su nuevo contexto escolar. 


\section{Metodología}

Con la finalidad de resolver la hipótesis y la pregunta de investigación, la presente investigación se sustentó en un método de investigación cualitativo, descriptivo, con el objetivo de documentar las experiencias subjetivas del entorno y de los niños en situación de movilidad humana que se integraron al contexto educativo ecuatoriano. De esta forma, fue posible identificar, documentadamente, la relación existente entre la adaptación social, como variable dependiente, y la resolución o no del proceso de duelo ocasionado por la situación de movilidad humana.

Considerando los factores previamente detallados, se seleccionó como población a niñas y niños integrados escolarmente en los niveles de educación general básica (EGB) elemental y media, de procedencia venezolana, que llegaron al Ecuador entre el año 2018 y 2019, y que migraron por la situación de crisis que vive Venezuela. Además, tomando en cuenta que Quito es una de las ciudades con mayor asentamiento de población venezolana, se definió como zona de influencia un barrio del norte de la capital donde existen numerosas familias venezolanas residiendo y que envían a niños y niñas a la escuela. Finalmente, para identificar a quiénes han tenido dificultades con su proceso de adaptación social a su nuevo contexto educativo como síntoma de posibles duelos no resueltos, se incorporó al estudio a niños y niñas que fueron derivados por sus instituciones educativas a centros de apoyo psicológico externo.

De esta forma, el presente estudio incluyó 2 unidades de información, 2 niñas y 1 niño: $\mathrm{N} 1$, niña de 10 años, que cursa el $7 \mathrm{mo}$. año de educación general básica (EGB) y que fue remitida por dificultades en su proceso de adaptación social al entorno escolar. N2, niña de 7 años, quien se encuentra en 3er. año de EGB, y que fue remitida por presentar episodios repetitivos de agresión física y verbal hacia sus compañeros, conductas de oposición y desobediencia a la autoridad. N3, niño de 7 años, que está en 3er. año de EGB 
Instituto Internacional de Investigación y Desarrollo Tecnológico Educativo INDTEC, C.A.

DOI: https://doi.org/10.29394/Scientific.issn.2542-2987.2020.5.E.2.48-69

OAI-PMH: http://www.indteca.com/ojs/index.php/Revista_Scientific/oai

\section{Artículo Original / Original Article}

y que fue remitido por no cumplir con las normas establecidas en el aula y agresión física y verbal hacia sus pares.

A partir de la definición de la población y para la recolección de las experiencias subjetivas, se utilizaron las siguientes técnicas e instrumentos de investigación:

1. Genograma (aplicado a las familias): a través del cual se obtuvo información de las estructuras y dinámicas familiares, tanto de aquellas previo al proceso migratorio, como de las conformadas una vez se realizó el asentamiento en el Ecuador.

2. Entrevistas semiestructuradas dirigidas a las familias: estas permitieron identificar las experiencias del proceso migratorio, antes, durante y después del mismo, y las vivencias y experiencias del proceso de inclusión social y adaptación al contexto educativo, tanto por parte del niño o la niña como de la propia familia. A su vez, este instrumento permitió conocer sobre el reconocimiento o no de signos de dificultades conductuales en el entorno familiar y su posible trayectoria.

3. Entrevistas semiestructuradas dirigidas a niñas/niño: este instrumento permitió conocer la experiencia vivida por el niño y las niñas durante el proceso migratorio, identificar señales del proceso de resolución del duelo, plantear sus propias vivencias con respecto a la dinámica y estructura familia, así como conocer, de primera mano, cómo se vive el proceso de inclusión educativa al nuevo contexto.

4. Cuestionario dirigido a docentes: mismos que permitieron identificar los signos o presuntas manifestaciones de las dificultades reportadas para la derivación psicológica y ahondar sobre la forma en la que se potenció o no, desde la escuela, el proceso de inclusión y adaptación social al nuevo entorno educativo.

Las mencionadas técnicas fueron aplicadas en la población objetivo del 
presente estudio durante un mes, tiempo en el que se recibió la información desde las distintas fuentes y fue posible interactuar con el niño y las niñas en el espacio terapéutico derivado. Una vez obtenidos los datos requeridos, los mismos fueron analizados a la luz del enfoque de la Terapia Familia Sistémica, a partir de los siguientes indicadores relacionados con las variables planteadas para el presente estudio: estructura y dinámica familiar previas a la migración y actuales, experiencias antes, durante y después del proceso migratorio, signos de dificultades conductuales, adaptación e inclusión de los estudiantes y participación de las familias en las actividades de la institución educativa.

\section{Resultados}

La presente investigación ha identificado los siguientes hallazgos con respecto a la incidencia de la dinámica familiar en la resolución del duelo y esta, a su vez, en el proceso de adaptación social de niños y niñas en situación de movilidad humana, desde Venezuela, a su nuevo contexto educativo.

Es posible identificar que a N1 se le informó sobre el viaje, así como el lugar de destino y se le brindó la explicación sobre lo que motivaba la migración. N1 menciona: mi mamá me dijo vamos a ir a Ecuador porque la economía en Venezuela es muy baja y mi mamá tenía asma y no había los medicamentos necesarios. Así mismo, la familia de N1 manifiesta que la vida en Venezuela era buena antes de la crisis.

En el mismo sentido, a N2 también le comunicaron sobre la decisión de viajar y al lugar al que llegarían. Sin embargo, a diferencia del caso anterior, se considera que la inseguridad y la falta de alimentos en Venezuela fue la que les obligó a salir de su país de origen.

En cuanto al tercer caso, se informa que N3 también fue comunicado sobre la decisión de viajar y el lugar de destino y N3 refiere que: mi tía me dijo que vamos a viajar a Ecuador, porque en Venezuela había saqueos, no había dinero. En definitiva, con respecto a la toma de decisión sobre el hecho de 
migrar, se evidencia que en los tres casos las niñas y el niño no participaron de la decisión y solo fueron informados. Además, en dos de los tres casos se evidencia que la decisión se encuentra forzada por situaciones extremas y de crisis que afectaba tanto a los niños como a sus familias. Adicionalmente, es importante destacar que, en los tres casos, se conoce de antemano el lugar de destino, el cual es transmitido a los niños.

A partir del proceso migratorio y asentamiento en el lugar de destino, la estructura familiar nuclear de los tres casos se mantiene, ya que actualmente todos ellos continúan viviendo con mamá, papá y hermanos. Sin embargo, manifiestan que existen una serie de variaciones en relación con la dinámica familiar que actualmente viven los niños. Esta se expresa, en primer lugar, por la distancia con ciertos miembros de la familia extendida con quienes compartían y hecho que entristece a los niños.

Por ejemplo, en el caso de N2, la familia menciona que la abuela y tío paterno de mi hija, con quienes convivíamos, se quedaron en Venezuela. Hay mucha nostalgia, por lo que siempre hablamos por teléfono o (hacemos) video llamadas. En este mismo sentido, como segunda modificación de la dinámica familiar, es posible identificar que las familias y los niños cuentan con poco tiempo para la convivencia y compartir actividades ya que cumplen, de manera extendida, con actividades laborales.

En este aspecto, N1 dice durante la entrevista que: "la vida en Ecuador es regular, porque mi papá trabaja mucho y no me da el cariño que quisiera". Así mismo, la familia de N2 indica que: las relaciones entre nosotros actualmente son agitadas, compartimos poco, no hay tiempo de esparcimiento o distracción porque tenemos que trabajar mucho.

Por tanto, es posible identificar que, si bien las estructuras familiares nucleares se mantienen después del proceso migratorio y que las familias y los niños siguen calificando como buenas las relaciones al interior de las mismas, la distancia con la familia extendida y los horarios de trabajo de las 
OAI-PMH: http://www.indteca.com/ojs/index.php/Revista_Scientific/oai

Artículo Original / Original Article

personas adultas han modificado las dinámicas familiares de los tres casos.

Con respecto a la respuesta emocional que enfrentan los niños ante la pérdida de los vínculos producto del proceso migratorio, es importante considerar que el duelo es un proceso natural que se experimenta frente a cualquier ruptura o separación.

En este sentido es posible identificar, a través de la información brindada, que N1, a pesar de haber llegado al país hace 2 años, aún se encuentra experimentando sentimientos y emociones vinculadas a la tristeza. Así lo refiere su familia, quien menciona que: la niña llora porque se siente triste de haber dejado su país.

En el caso de N2, se informa que constantemente se siente triste y extraña a su abuela paterna. Por último, en el caso de N3 se refiere que se encuentra constantemente aburrido y su familia menciona que: (se ha) notado al niño rebelde, no desea realizar las actividades de la escuela y la casa. Se le nota un poco apagado. Por tanto, se evidencia que en los tres casos existen sentimientos de tristeza propios del proceso de duelo que experimentan tras abandonar su país y separarse de sus familias extendidas. Sin embargo, se debe evidenciar que el tiempo transcurrido desde la llegada al Ecuador, en los tres casos, es de más de un año, lo que puede indicar que los tres niños requieren apoyo para la resolución de su duelo.

Con respecto a este proceso, los 3 casos son derivados desde la escuela a centros de atención psicológica, considerando que han presentado episodios de agresión física y verbal a sus pares, así como rasgos que se describen como enfrentamientos a la autoridad, incumplimiento de la normativa y aislamiento de su grupo etario. En el caso de N1 el reporte de la docente manifiesta que: han existido episodios repetitivos de agresión verbal de la estudiante a sus compañeros, la niña no muestra interés por relacionarse y compartir con sus pares, ni disposición para realizar trabajos en grupo, desobedece las disposiciones que se le da. 
Con respecto a la adaptación social, N1 indica que le gusta su escuela y que siente cariño por su actual maestra, así como por su grupo de amigas. Adicionalmente, menciona que no tiene muy buena relación con ciertos niños de su grupo, ya que: (estos) me caen mal porque me dicen cosas feas, no me dejan participar, me aíslan. Al respecto, la familia de N1 manifiesta que a la niña le gusta su escuela, pero se queja del trato de sus compañeros.

En el caso de N2, la niña también afirma sentirse contenta en su actual escuela e indica que no tiene muchos amigos, ya que en ocasiones la excluyen de los grupos, menciona que: la relación con mis compañeros es mala, sólo tengo 2 amigas que me tratan bien, les pregunto a los demás niños si puedo jugar con ellos y me dicen que no. Así también la familia de N2 señala que: la niña no quiere trabajar, dice que no tiene amigos, le cuesta adaptarse.

Finalmente, en el caso de N3, el niño menciona que tiene amigos con los que le gusta jugar, sin embargo, menciona que cuando llegué un niño me quitó mi colación y me pegó. La familia de N3 argumentó que a mi hijo le gusta la escuela, pero existen compañeros que no lo tratan bien, lo que le hace sentir mal. A partir de estas puntualizaciones, con respecto a la relación entre el sistema familia y el sistema escuela, se puede identificar que existe información contradictoria. Esto dado que la escuela remite dificultades en la adaptación social de los niños en situación de movilidad humano y no emite ninguna información sobre las agresiones que manifiestan los niños y sus familias. Por el contrario, los tres niños y sus familias expresan que, si bien les gusta la escuela, han recibido tratos inadecuados por parte de sus compañeras y que tiene pocos amigos o son aislados por su grupo de pares.

Con respecto a este tema, la docente de N1 refiere que no se realizó ninguna acción específica para recibir a la niña al momento de su ingreso a la escuela y que tampoco se brindó información detallada a la estudiante o a la familia acerca de su nuevo contexto educativo o cómo funciona la escuela, conforme menciona la docente: cuando N1 se integró al aula no se generó 
OAI-PMH: http://www.indteca.com/ojs/index.php/Revista_Scientific/oai

\section{Artículo Original / Original Article}

ninguna acción para el recibimiento de sus compañeros, no se le brindó información detallada ni a ella ni a su familia acerca de la institución, (ni sobre) sus normas de convivencia (o) su personal.

En el caso de N2, en su aula se recibió a la niña con aplausos, pero conforme refiere la docente tampoco se realizó un proceso de inducción con la niña o su familia. Al respecto, la familia de N2 menciona que: no hubo adaptación, el ritmo de la profe era muy lento porque mi hija ya sabía sumar, restar, leer y escribir de corrido, los niños la ven como una persona diferente y no la aceptan. Por último, el docente de N3 menciona que: (sus) compañeros lo recibieron con un aplauso y le dieron la bienvenida, así mismo se menciona que se brindó información detallada a la familia y al niño sobre la nueva escuela. Sin embargo, en contradicción, la familia menciona que: no existió un buen proceso de integración, hay compañeros que lo molestan y le ponen apodos.

Por este motivo, se puede identificar que las tres familias coinciden que existen diferencias en los sistemas educativos de Venezuela y Ecuador a las que han tenido que adaptarse y que para este proceso de adaptación hizo falta generar acciones que permitan un proceso real de inclusión educativa. En definitiva, estas contradicciones permiten evidenciar que no existe una dinámica entre el sistema escolar y el sistema familiar, situación que dificulta el proceso de adaptación social e inclusión educativa.

\section{Conclusiones}

Con respecto a la presente investigación, en la que se aplicó un método cualitativo descriptivo para identificar las experiencias subjetivas en cuanto a la resolución del duelo producto de la situación de movilidad humana y a la adaptación social al nuevo contexto educativo de niños y niñas, se pudo identificar que, aunque la población objeto de estudio migró y actualmente vive con la misma estructura familiar nuclear con la que vivía en Venezuela, las 
condiciones en el país de destino han modificado las dinámicas familiares. Esto considerando que las personas adultas de estos núcleos familiares están expuestas a jornadas laborales extendidas y a situaciones en las que experimentan preocupación, agobio, tensión y tristeza por la condición económica producto del proceso migratorio.

Estos cambios en la dinámica familiar han afectado directamente en el tiempo que sus miembros comparten actividades, generan espacios de diálogo, se acompañan en los procesos de duelo por la situación de movilidad y se apoyan en los respectivos procesos de adaptación social al nuevo entorno.

Al cambio y modificación de la dinámica familiar y a la sensación de soledad producto de la separación de su entorno de origen y su familia extendida, se suma el hecho que niños y niñas no fueron parte del proceso de toma de decisiones sobre la migración. Sobre este tema, ellos conocen las razones que motivó a sus familias a migrar, sin embargo, solo fueron informados sobre la decisión tomada por los adultos que, en estos casos, incluye conocer de forma anticipada el país de destino. Este hecho provoca una sensación de obligación ante el hecho de migrar.

Con respecto a la adaptación social al entorno escolar, es posible concluir que la escuela no ha implementado acciones afirmativas para que niños y niñas en situación de movilidad puedan, efectivamente, sentirse incluidos al nuevo contexto educativo. Este argumento se sustenta en que las instituciones educativas solo garantizaron el acceso educativo, a través de la matrícula e ingreso a clases, sin generar acciones que permitan a estudiantes y sus familias tener un conocimiento básico sobre la nueva realidad educativa o sentirse acogidos en el nuevo entorno social. Adicionalmente, las instituciones educativas buscan que las familias sean quienes se acerquen en cuanto son llamados, sin considerar que sus horarios laborales hacen imposible que esto suceda. 
OAI-PMH: http://www.indteca.com/ojs/index.php/Revista_Scientific/oai

Artículo Original / Original Article

Finalmente, se remite a los niños, sujeto de este estudio, a centros de acompañamiento psicológico, argumentando dificultades en adaptación social a su entorno escolar. Este hecho no considera que las familias y los propios niños reportan que han sido víctimas de agresiones verbales y físicas por parte de sus pares, hechos que les han provocado aislamiento, responder con agresión y no tener amigos. Así mismo, la falta de procesos de inclusión puede haber determinado en el incumplimiento de normas por desconocimiento del sistema y cómo funciona. En definitiva, la derivación de estas niñas y niños por problemas de adaptación social se la realiza con desconocimiento del contexto e ignorando las necesidades básicas de quienes están atravesando un proceso de migración.

En este sentido, es posible concluir que, si bien el grupo de niños objeto de este estudio presenta ciertos rasgos que evidencia dificultades de adaptación social, este hecho se encuentra mediado por la situación de movilidad humana y la falta de estrategias del entorno escolar para facilitar la inclusión educativa. Por otro lado, se evidencia también que la resolución del duelo por el hecho de migrar se encuentra aún en proceso, para lo cual las niñas y los niños requieren contar con el apoyo de su contexto, que en este caso lo conforman los sistemas familiar y escolar. Por tanto, es importante destacar que la escuela debe reconocer que la situación de movilidad provoca un proceso de duelo, en el que este sistema debe involucrarse activamente y a través del desarrollo de acciones afirmativas para apoyar una efectiva inclusión educativa.

Para que los sistemas escolar y familiar apoyen a niñas y niños en situación de movilidad humana en su proceso de resolución del duelo, se debe empezar por reconocer que niñas y niños también experimentan sentimientos de tristeza, frustración, enojo, que interfieren en el proceso de adaptación social al nuevo entorno. En este sentido, la escuela debe generar acciones de acogida a los niños y sus familias en situación de movilidad humana que, 
además de eliminar comportamientos de discriminación y xenofobia en docentes y estudiantes, permitan contar con información sobre normas, funcionamiento del sistema educativo y del nuevo entorno.

Además, para que exista una coordinación efectiva entre los dos sistemas, es la escuela quien debe reconocer las condiciones diversas que median el contexto de las personas que migran, por lo que es la institución educativa quien debe adaptarse para incluir a la población en situación de movilidad. Por último, es indispensable que el sistema familiar y escolar puedan acompañar en conjunto para la resolución del duelo de niñas y niños y para esto se debe empezar por el reconocimiento de las necesidades específicas de quienes, a esta edad y sin ser parte de la toma de decisiones sobre migrar, deben dejar su país de origen y enfrentarse a un nuevo contexto.

En conclusión, la hipótesis que orientó el presente estudio no pudo ser confirmada a través de los casos objeto de investigación. Esto considerando que las dificultades de adaptación de los niños en situación de movilidad, no se encuentra relacionada con la dinámica familiar y como esta favorece en la resolución del duelo. Por tanto, con respecto a la pregunta de investigación, se puede considerar que lo que afecta el proceso de adaptación social de las niñas y niños en situación de movilidad humana, es la dinámica del sistema escolar y la coordinación entre los sistemas escuela y familia para apoyar en la resolución de su duelo.

\section{Referencias}

Atxotegui, J. (2000). Los duelos de la migración: una aproximación psicopatológica y psicosocial. ISBN: 84-7290-152-1. España: Editores Bellaterra.

Ceja, I., Villarreal, M., Fonseca, Á., Avetta, L., Correa, T., Fernández, L., ... Cabezas, G. (2020). Voces y experiencias de la niñez y adolescencia venezolana migrante en Brasil, Colombia, Ecuador y Perú. 1ra. 
edición, ISBN: 978-987-722-578-5. Buenos Aires, Argentina: CLACSO. Colegio Oficial de Psicólogos de Madrid (2016). Guía para la intervención psicológica con inmigrantes y refugiados. ISBN: 978-84-87556-661. Madrid, España: Colegio Oficial de Psicólogos de Madrid.

Coronel, F. (2013). Efectos de la migración en el proceso de aprendizajeenseñanza y su tratamiento desde la escuela. Revista de Investigación Educativa, 6(1), 57-77, e-ISSN: 1997-4043. Recuperado de: http://www.scielo.org.bo/pdf/rieiii/v6n1/v6n1a04.pdf

González, V (2005a,b). El duelo migratorio. Trabajo Social, (7), 77-97, ISSN: 0123-4986, e-ISSN: 2256-5493. Recuperado de:

https://revistas.unal.edu.co/index.php/tsocial/article/view/8477

Hernández, C. (2019). El Proceso de Enseñar y Aprender: Indagación desde el Contexto Educativo. Revista Scientific, 4(12), 254-274, eISSN: 2542-2987. Recuperado de:

https://doi.org/10.29394/Scientific.issn.2542-2987.2019.4.12.13.254$\underline{274}$

Kreuz, A., Casas, C., Aguilar, I., \& Carbó, M. (2009). La convivencia escolar desde el enfoque sistémico. El niño en la encrucijada entre sociedad, familia y escuela. Informació psicològica, (95), 46-61, eISSN: 0214-347X. Recuperado de:

https://dialnet.unirioja.es/servlet/articulo?codigo $=3039000$

Papalia, D., Wendkos, S., \& Duskin, R. (2009). Psicología del Desarrollo: de la infancia a la adolescencia. Undécima Edición, ISBN: 978-970-106889-2. México, D.F.: McGraw-Hill / Interamericana Editores, S.A. de C.V.

Sánchez, A. (2018). Los derechos de las personas en situación de movilidad humana en Quito (DMQ): percepciones de los distintos actores involucrados a partir de la inmigración venezolana. Tesis. Quito, Ecuador: Pontificia Universidad Católica del Ecuador. 
Instituto Internacional de Investigación y Desarrollo Tecnológico Educativo INDTEC, C.A.

DOI: https://doi.org/10.29394/Scientific.issn.2542-2987.2020.5.E.2.48-69

OAI-PMH: http://www.indteca.com/ojs/index.php/Revista_Scientific/oai

Artículo Original / Original Article

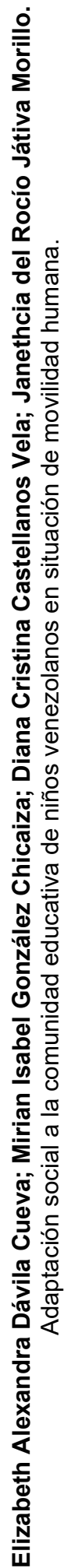

Recuperado de: http://repositorio.puce.edu.ec/handle/22000/14939 Suarez, M. (2010). El Genograma: herramienta para el estudio y abordaje de la familia. Revista Médica La Paz, 16(1), 53-57, e-ISSN: 1726-8958. Recuperado de:

http://www.scielo.org.bo/scielo.php?script=sci arttext\&pid=S1726$\underline{89582010000100010}$ 
OAI-PMH: http://www.indteca.com/ojs/index.php/Revista_Scientific/oai

Artículo Original / Original Article

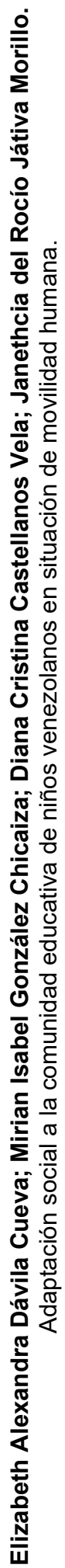

\section{Elizabeth Alexandra Dávila Cueva \\ e-mail: eadavila.mpp@uisek.edu.ec}

Nacida en Quito, Ecuador, el 11 de agosto del año 1988.

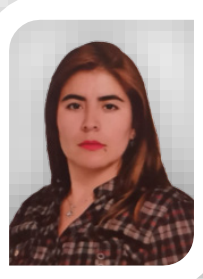

Estudié Psicología Clínica en la Universidad Central del Ecuador (UCE); culminé mis estudios profesionales en la Maestría de Psicología Mención Psicoterapia en la Universidad Internacional SEK (UISEK); actualmente me encuentro laborando como Analista del Departamento de Consejería Estudiantil adscrito al Ministerio de Educación del Ecuador. 


\section{Artículo Original / Original Article}

\section{Mirian Isabel González Chicaiza}

e-mail: migonzalez.mpp@uisek.edu.ec

Nacida en Quito, Ecuador, el 3 de octubre del año 1992.

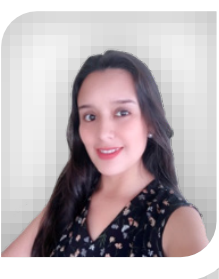

Estudié en la Universidad Central del Ecuador (UCE); obtuve el título de Licenciada en Ciencias de la Educación con mención en Psicología Educativa y Orientación; mis estudios de cuarto nivel los realicé en la Universidad Internacional SEK (UISEK), obteniendo el título de Magister en Psicología con mención en Psicoterapia; me desempeñé como Psicóloga Educativa adscrita al Ministerio de Educación del Ecuador; actualmente soy Analista del Departamento de Consejería Estudiantil. 
OAI-PMH: http://www.indteca.com/ojs/index.php/Revista_Scientific/oai

\section{Artículo Original / Original Article}

\section{Diana Cristina Castellanos Vela}

e-mail: diana.castellanosv@gmail.com

Nacida en Quito, Ecuador, el 11 de julio del año 1985.

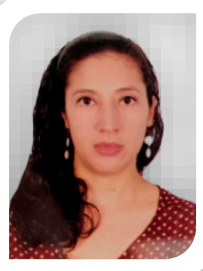

Psicóloga Educativa por la Pontificia Universidad Católica del Ecuador (PUCE); magíster en Gestión del Talento Humano por la Universidad Tecnológica Equinoccial (UTE); me he desempeñado como psicóloga de instituciones educativas, como analista educativa en el Ministerio de Educación y en atención a personas privadas de libertad del Ministerio de Justicia, Derechos Humanos y Cultos; así como en la política de atención a primera infancia en la Secretaría del Plan Toda una Vida; Actualmente me desempeño como docente universitaria y gestora en proyectos comunitarios. 


\section{Artículo Original / Original Article}

\section{Janethcia del Rocío Játiva Morillo}

e-mail: rocio.jativa@uisek.edu.ec

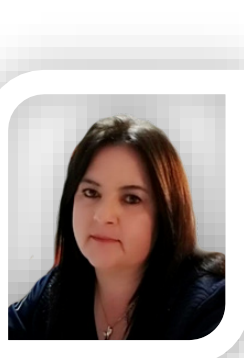

Nacida en Quito, Ecuador, el 15 de marzo del año 1968.

Licenciada en Psicología de la Universidad Central del

Ecuador (UCE); Doctora en Administración por la Universidad Escuela Politécnica Javeriana (ESPOJ);

Magister en Intervención y Terapia Familiar Sistémica por la Universidad Politécnica Salesiana (UPS); Experiencia en el ámbito educativo, docente y psicóloga de Institutos Militares, práctica clínica a favor de la niñez, adolescencia y de grupos de atención prioritaria en el Ministerio de Inclusión Económica y Social (MIES) y el Ministerio de Justicia, Derechos Humanos y Cultos (MJDHC); también cuento con experiencia en Formación Inicial de Jueces, Fiscales y Defensores Públicos, como Subdirectora de la Escuela de la Función Judicial, consulta privada y docente de maestría en la Universidad Internacional SEK (UISEK).

El contenido de este manuscrito se difunde bajo una Licencia de Creative Commons ReconocimientoNoComercial-Compartirlgual 4.0 Internacional 